\title{
Aoristo)))))
}

International Journal of Phenomenology, Hermeneutics and Metaphysics

\section{Viaggio verso la fondazione della morale in Edmund Husserl}

\author{
Journey towards the foundation of morality in Edmund \\ Husserl
}

\author{
Prof.essa. Dr.essa. Maria Concetta Ripullo \\ Università degli Studi di Messina'
}

\section{RIASSUNTO}

La fenomenologia husserliana è attraversata da una tensione etica indirizzata inizialmente a definirne il rigore "scientifico"; nelle fasi successive, del pensiero del Maestro, l'etica diventa tema specifico di riflessione all'interno di una "fenomenologia della ragione pratica" il cui riferimento essenziale è costituito da un valore intenzionato nel quale io ho, ed ogni io ha, l'origine. Questa assoluta Originarietà include "i modi di coscienza empatici e comprensivi" ed apre ad un eidos comune agli esseri umani come persone, eidos la cui evidenza fattuale è l'amore (personale ed etico) come "disposizione durevole verso gli altri verso l'intera umanità", questa è un'idea che si trova nell'essere umano come formata "preontologicamente". La riflessione su questa assoluta originarietà, consente al Nostro di trovare un fondamento comune intersoggettivo, un'idea che in quanto ontologicamente fondata può diventare, come Telos, trainante per la volontà: "scopo di tutti gli scopi... idea dell'essere soggettivo perfetto all'interno di una comunità totale intersoggettiva perfetta all'infinito". Husserl denomina la teleologia come "forma di tutte le forme" nella quale si trova la soggettività.

\section{PAROLE-CHIAVE}

Valore; Persona; Amore; Intersoggettività; Telos

\section{ABSTRACT}

Husserlian phenomenology is crossed by an ethical tension initially aimed at defining its "scientific" rigor; in the subsequent phases, of the Master's thought, ethics becomes a specific theme of reflection within a "phenomenology of practical reason" whose essential reference is constituted by an intentional value in which I have, and every I has, the origin. This absolute Originarity includes

\footnotetext{
${ }^{1}$ E-mail: mariaconcetta.ripullo@gmail.com
} 


\title{
Aoristo)))))
}

International Journal of Phenomenology, Hermeneutics and Metaphysics

\begin{abstract}
"empathic and understanding modes of conscience" and opens to an eidos common to human beings as persons, eidos whose factual evidence is love (personal and ethical) as a "lasting disposition towards others towards whole humanity", this is an idea that is found in the human being as formed" preontologically". The reflection on this absolute originality allows our to find a common intersubjective foundation, an idea which, as ontologically founded, can become, like Telos, a driving force for the will: "aim of all purposes idea of the subjective being perfect all 'interior of a total infinity perfect intersubjective community". Husserl calls teleology the "form of all forms" in which subjectivity is found.
\end{abstract}

\section{KEYWORDS}

Value; Person; Love; Intersubjectivity; Telos

La ricerca in materia pratico-etica di Husserl si caratterizza anzitutto come fenomenologia degli atti affettivi o valutativi. Si tratta di chiarire come veniamo a conoscenza delle leggi che regolano tale materia e quale sia la natura di esse. L'indagine prende ad esame, primariamente, l'analogia tra gli atti teoretici o conoscitivi e gli atti affettivi o valutativi.

Giudicare, afferma Husserl, significa innanzitutto presumere, intendere, credere che qualcosa sia o non sia. Ogni atto conoscitivo è diretto su qualcosa: in ogni giudizio distinguiamo l'atto di intendere e l'oggetto inteso.

Anche l'atto affettivo o valutativo presenta una struttura analoga: distinguiamo il desiderio dal desiderato, il volere dal voluto ecc.

Secondo Husserl, la coscienza è un campo di presupposizioni, questo vale sia per gli atti conoscitivi che per quelli affettivi e pratico-etici, di fatto l'atto affettivo è un presumere, un credere qualcosa come buono, piacevole, spiacevole, cattivo, bello ecc. La differenza fondamentale tra gli atti affettivi e quelli conoscitivi è che questi ultimi portano a manifestazione un oggetto, nelle Ricerche Logiche sono definiti "atti oggettivanti".

Gli atti affettivi, invece, non portano a manifestazione alcun oggetto, ma si dirigono su un oggetto già inteso o rappresentato, essi vengono definiti nella stessa opera "atti non oggettivanti": non riguardano l' esistenza di un oggetto ma ci dicono che un determinato oggetto è buono o cattivo, piacevole o spiacevole, l'atto affettivo è tale perché è intenzionale ossia si dirige, comunque, su qualcosa d'altro da sé, si dirige direttamente sull'oggetto fondante (su un oggetto gradevole o piacevole già portato a manifestazione dall' atto conoscitivo), ma indirettamente si dirige "sul piacevole" che è al di là dell'oggetto rappresentato. Il "piacevole" è la materia propria degli atti affettivi, è un oggetto sui generis che Husserl chiama "valore" (Wert), "[...] gli atti valutativi divengono coscienti manifestazioni di valore".

"Valore -precisa Husserl - non è un ente [...] ma appartiene ad un'altra dimensione"(HUSSERL, 1968, p.179)

La fondazione dell'atto affettivo su quello oggettivante non ha nulla di casuale, sia esso volitivo, valutativo ecc., si da' sempre come un atto complesso, cioè con una 


\section{Aoristo)))))}

\section{International Journal of Phenomenology, Hermeneutics and Metaphysics}

pluralità di qualità intenzionali: infatti possiamo distinguere una intenzione percettiva, una rappresentativa e una intenzione affettiva; questo" intreccio" necessario tra coscienza dossica e coscienza affettiva comporta il riconoscimento dell'impossibilità, rimanendo nella sfera dell'affettività, di astrarre dall'intelletto.

Se l'intelletto può fare a meno del sentimento, il contrario è impossibile; Husserl afferma che l'intelletto esercita necessariamente le sue funzioni anche nella sfera affettiva.

Si può sostenere, perciò, che ogni atto pratico- etico ha il suo lato razionale , ciò significa che la sfera affettiva non è qualcosa di eterogeneo rispetto a quella teoreticoconoscitiva.

Per questo motivo Husserl parla di predominio dell'elemento logico o di universalità del fattore logico, grazie a ciò è possibile istituire, accanto o parallelamente ad una logica del giudizio, anche una logica del sentimento (questa visione sarà capovolta da Scheler).

Un'etica scientifica è la descrizione di questa logica. Io posso studiare l'esperienza, le intuizioni e le operazioni costitutive, e posso farlo perché gli universali non sono astrazioni, ma tipicità e strutture essenziali a cui corrispondono sempre gli Erlebnisse.

Le forme logiche sono correlative alle operazioni, come gli oggetti sono correlativi al soggetto, ma nelle forme logiche c'è di più di quella che noi consideriamo una realtà: c'è un'idealità, un significato, essi non sono nella dimensione naturalistica, ma nella sua verità, nel suo orizzonte, nel suo telos razionale.

Caratteristico dell'essere umano è che ciò che egli sente, percepisce o si rappresenta, supera il dato naturale, tende al tipico e all'universale, alla forma: l'uomo tende a rappresentarsi il significato della sua vita e del mondo che porta in sé.

$E^{\prime}$ questa l'esigenza, tutta spirituale, che induce Husserl a manifestare una tensione etica che attraversa tutta la sua copiosa opera, esprimendo l'anelito a superare l'immanenza cui l'obiettivismo delle scienze incatena l'uomo.

Già nella Introduzione all'etica (1920-1924) (HUSSERL, 2019, p.24) si possono trovare gli spunti per i quali l'etica diviene tema specifico di riflessione all'interno di una "Fenomenologia della ragione pratica" il cui riferimento essenziale (non formale come in Kant) è costituito da un valore scelto, non di carattere puramente psicologico, quindi soggettivo, ma tendente a realizzare una sempre più alta umanità tramite l'affermazione dei valori di dignità ed autonomia della vita noematica delle persone, giusta l'affermazione dello stesso Husserl (Monadologia, 1930) (ALES BELLO, 1985, p.77).

La coscienza del dovere trova giustificazione nella rispondenza del volere del soggetto ad un valore originario immanente all'io. L'attenzione adesso si rivolge al significato che Husserl attribuisce al soggetto . In Idee per una fenomenologia pura e un filosofia fenomenologica II afferma: 


\section{Aoristo)))))}

International Journal of Phenomenology, Hermeneutics and Metaphysics

Essere soggetto significa in tutto e per tutto e niente altro che essere attivo; quindi l'essere soggetto e l'essere attivo coincidono, così come coincidono l'essere oggetto per un soggetto e l'essere prodotto di un'azione. Nulla c'è prima della azione: l'inizio della storia del soggetto non è un fatto ma è un atto (HUSSERL, 2002, p. 251-253).

Essere soggetto significa progredire necessariamente da azione in azione, dal prodotto di un'azione a nuovi prodotti, in quanto appartiene all'essenza dell'agire l'avere un fine. Ogni realizzazione richiede nuovi fini in una connessione infinita.

Qui è evidente come il Nostro, con la sua analisi, sostenga il valore etico della dignità della vita della persona.

Husserl, nella stessa opera, prende in esame la costituzione dell' io personale e scrive: "Il corso dei vissuti della coscienza pura è necessariamente un processo di sviluppo, lungo il quale l'io puro deve assumere la forma appercettiva dell'io personale [...] la persona è il soggetto delle facoltà $[. .$.$] facoltà che io conosco sulla$ base del loro modo di esperienza. L'io personale è l'io uomo; appercepisco me stesso come un uomo nel contesto umano, trovando numerosi spunti per osservare il mio proprio comportamento, imparo a conoscermi come una realtà personale.

Un uomo non si conosce, non sa che cos'è, impara a conoscersi. L'esperienza di sé, l'appercezione di sé si amplia costantemente. Quindi l'imparare a conoscersi è la stessa cosa della costituzione del sé [...] la peculiarità del soggetto spirituale sta nel fatto che in esso si fa avanti l'appercezione "io", nella quale il soggetto è oggetto. Bisogna distinguere, dunque, io che sono dal lato del soggetto, e io che sono quale obiectum per me, rappresentato, intenzionato nell'io-sono: il me (das Mich): si intende qui la persona costituita per me, l'io, che è presente alla coscienza come sé. [...] L'io spirituale può essere come un organismo di facoltà $[. .$.$] La facoltà è una potenzialità$ positiva che volta per volta, si attualizza, che è sempre pronta a trapassare in attività"(HUSSERL, 2002, p.253).

Nella genesi originaria, l'io persona non si costituisce soltanto come una persona determinata dagli impulsi, da istinti originari, passiva nei loro confronti, ma anche come un io superiore, autonomo, che agisce liberamente, guidato da motivi razionali.

"Le abitudini si sviluppano sia per il comportamento originariamente istintivo , sia per il comportamento libero [...]. Il fatto di lasciarsi determinare da un motivo di valore e di resistere a una pulsione fonda una tendenza [...]. Qui l'abitudine, s'intreccia con la libera motivazione. Quando agisco liberamente aderisco sì ad una abitudine, ma sono anche libero in quanto aderisco ai motivi, alla ragione nelle sue libere decisioni". Ciò che agisce come motivo porta in sé numerose implicazioni intenzionali: a) motivazioni a risalire al senso; b) ad aderire alla verificazione della verità stessa; c) a lasciarsi determinare, sulla base dell'autentica ragione, dalla verità"(HUSSERL, 2002, p. 267). L'autonomia della ragione, la libertà del soggetto personale consiste in questo: io non aderisco passivamente agli influssi estranei, agisco liberamente solo nella modalità della ragione. 


\title{
Aoristo)))))
}

International Journal of Phenomenology, Hermeneutics and Metaphysics

All'inizio dell'esperienza non c'è ancora un sé costituito, il sé è completamente nascosto a sé e agli altri. La fenomenologia trascendentale è una scienza della costituzione, del come l'io genera, ciò non vuol dire che devono essere costituite solo le forme o le essenze: la costituzione riguarda anche la cosa fisica, la psiche, gli altri esseri umani e quindi, in questo senso, tutto ciò che si dà nell'esperienza del soggetto, nell' Erlebnis.

Quando Husserl dice che la fenomenologia è trascendentale e non empirica vuol dire che la fenomenologia non assume gli oggetti reali, empirici, già fatti, così come essi si danno prima dell'epoché (della sospensione del giudizio ) della riduzione fenomenologica, nel loro piano mondano. L'esigenza fondativa che è essenziale nell'analisi fenomenologica, emerge anche in questo caso per affermare il come del ritorno alle "cose stesse".

E. Paci, nell'ambito della interpretazione delle Meditazioni Cartesiane di Husserl, rileva: "La nuova esistenza, l'esistenza che continuamente rinasce, è, per Husserl, una nuova infinita sfera dell'essere (Seinsphäre) che vive nel tempo il senso della vita come esperienza direzionale e teleologica della verità... questa esperienza si attua nella immanente forma temporale della corrente di coscienza" (PACI, 1961, p. 76-78).

Connesso alla costituzione dell'io emerge, pure, il problema della relazione tra gli Ego. Questa relazione introduce la dimensione intersoggettiva nella costruzione del mondo, così la comunità trascendentale del noi viene alla luce assieme agli altri soggetti.

Come strumento privilegiato di analisi antropologica, Husserl indica l'entropatia (il reciproco entro sentirsi, Einfühlung) tramite la quale riusciamo a cogliere ciò che si muove nella vita dell'altro relativamente ai suoi sentimenti, emozioni, al suo mondo di decisioni, volizioni, creatività.

"L'altro è il rispecchiamento di me stesso e, tuttavia, esso non è propriamente un rispecchiamento" (HUSSERL, 1994, p.51). L'esperienza dell'altro avviene tramite il suo corpo fisico, quindi indirettamente; l'altro in quanto soggetto, non può mai essere presente in originale. Questa "intenzionalità indiretta ", che rende conpresente l'altro al darsi del suo corpo fisico, viene chiamata da Husserl "appresentazione" (Apprasentation: render conscio come con-presente). Tale appercezione per analogia non è un atto del pensiero, un sillogismo, ma è una sorta di" fondazione originaria" (Urstiftung) o "accoppiamento originario" (Paarung) (HUSSERL, 1994, p.51).

Il mondo e gli altri, quindi, sono "dentro" la coscienza, sono immanenti alla coscienza in quanto percepiti e sentiti; ma, da un altro punto di vista, cioè in se stessi, sono trascendenti in quanto mi accorgo che l'altro è diverso da me, è un alter ego. Attraverso l'entropatia, posso comunicare con lui e sentire la nostra comune umanità, ma non posso in alcun modo identificarmi con lui. Ales Bello in Husserl e il problema di Dio mette in risalto la doppia riduzione fenomenologica che avviene grazie alla percezione dell'altro:

\author{
Maria Concetta Ripullo \\ Toledo, v. 4, n'2 (2021) p. 153 162
}




\section{Aoristo)))))}

International Journal of Phenomenology, Hermeneutics and Metaphysics

[...] se il primo movimento è quello di evidenziazione della mia sfera di appartenenza, il secondo è la scoperta dell'empatia che mi permette di penetrare nell' altro, pur rimanendo quest'ultimo fondamentalmente estraneo e diverso da me quindi trascendente (ALES BELLO, 1985, p.37).

Sorge, così, il problema del rapporto tra immanenza e trascendenza nella sfera originaria che ha come polo l'io.

Nelle Meditazioni Cartesiane Husserl affronta questo problema che diventa una ricerca fenomenologica guidata dall'idea dell'altro da me. L'altro, che all'inizio è solo un correlato del cogito, diventa un filo conduttore trascendentale della ricerca.

Gli altri sono sentiti da me sia come oggetti (psicofisici) che come soggetti. Se sono soggetti avranno anche loro (per analogia) delle percezioni come le ho io in quanto soggetto; io ho un mio mondo e gli altri avranno ,allora, ciascuno un proprio mondo.

La Quinta Meditazione tende a esplicitare il senso dell'esperienza naturale, sociale e culturale in quanto "senso dell'esperienza che è per ognuno".

Husserl, per introdurre le lezioni "Sulla via verso una scienza della soggettività trascendentale", deve affrontare la questione del difficile equilibrio tra soggettività e mondo; esso dichiara che prima di iniziare con la riflessione sulle strutture del mondo dell'esperienza, è necessario effettuare "[...] un altro inizio: [...] debbo compiere meditazioni generali sullo scopo della conoscenza e della scienza [...]. Medito anche sulla visione intellettuale, sull'evidenza, mi chiarisco come la vita giudicativa possa diventare conoscitiva e possa giudicare se stessa; dovrò come, ciò che è identificabile nell'intellegibilità e nel giudizio, viene ad autodatità, quindi è dato attraverso l'evidenza apodittica [...], si instaura allora la teoria scientifica delle forme, il cui ambito è dato attraverso l'evidenza apodittica [...], pervengo alla distinzione fra cose, valori, beni. (Se) La logica formale era la logica delle proposizioni giudicative, (ora) consideriamo quelle valutative, rispettivamente desiderative e volitive, allora si delineano parallelamente i concetti della verità di giudizio (nasce la logica del giudizio come logica della verità ) verità relativa al valore: verità pratica. Così si dà non solo una scienza logica dei fatti, ma anche una logica dei valori reali e degli scopi" (ALES BELLO, 1985, p. 55).

La logica formale si allarga ad una logica sia delle proposizioni valutative, che delle verità di valore. "Il giudicare rimanda alla soggettività giudicante e "questa può avere anche carattere giudicativo - volitivo" quindi il formale della nuova sfera ha un universale altrettanto grande come il concetto di logica formale. " Infine si ottiene, dunque, la logica di un'umanità etica [...] la logica di uno sviluppo di un'umanità genuina nei gradi dei suoi prodotti e della sua vita produttiva" (ALES BELLO, 1985, p. 57-58).

L'indagine sistematica di tutte le teleologie che si possono trovare nel mondo empirico (dallo sviluppo degli organismi fino all'uomo) non si esaurisce nelle spiegazioni scientifico-naturalistiche. L'esigenza della fenomenologia (quella di ritornare alle "cose stesse", alla loro essenzialità) attuata con la riduzione 


\section{Aoristo)))))}

International Journal of Phenomenology, Hermeneutics and Metaphysics

trascendentale al soggetto, conduce, ora, alle questioni riguardo sia il fondamento della fatticità, ovvero riguardo il fatto considerato come sorgente di valori possibili e reali, sia riguardo la coscienza costitutiva. Il mondo, inteso come il complesso di ciò che è trascendente, non è messo in dubbio, ma esaminato nel suo costituirsi per noi. Qui si manifesta necessariamente una esigenza regressiva che conduce alle fonti della conoscenza, a ciò che sta dietro le formazioni culturali: è la ricerca dell'originario, dell" "Ur".

La realtà naturale e storica, il mondo- della- vita (Lebenswelt) circostante, trovano il loro fondamento giustificativo nell'ontologia del mondo- della- vita. Struttura, questa," antepredicativa" ed originaria, strettamente connessa con il modo di intendere la coscienza come "flusso " temporale, che permette di comprendere la storia e il suo significato. Se il passato è percepito nel presente come ricordo , la percezione si muove sempre verso un futuro e proprio in tal senso è appercezione.

La sfera del proprio è questo Kern (nucleo) complesso di percezioni e di non percezioni, di potenziale e di attuale.

Afferma Husserl: “L' Ego stesso c'è per se stesso in uno stato continuo di evidenza, in quanto costituente continuamente se stesso come esistente " (HUSSERL, 1994). Così l’Ego si afferma, contemporaneamente, come genetico e genealogico. "[...] Ogni io in quanto soggetto identico alla sua coscienza pura, è appercepibile come un qualche cosa che ha i suoi modi (che hanno una certa configurazione) di comportarsi nei confronti del suo mondo circostante, un suo modo determinato di farsi motivare da esso, attraverso modi attivi e passivi di comportamento; e chiunque sia giunto attraverso un certo sviluppo ad una certa maturità, appercepisce se stesso in questo modo, si trova nel mondo come una persona" (HUSSERL, 1994, p. 320).

L'uomo non può trovarsi vivo da solo: si trova vivo nel senso della natura, della socialità, della storia. Possiamo affermare che la fenomenologia integra l'uomo nella intenzionalità della vita temporale universale. Per altro la centralità della ricerca etica è l'io persona.

La fenomenologia, scrive Enzo Paci, è una "formazione " dell'essere umano nel senso dell'universo aperto. E' un'educazione continua che ci fa uscire dall'alienato, dal separato per introdurci alla vita relazionale.

Scoprendo il proprio e l'Alter ego, scopro in me un'esperienza reale, mia propria, dell'alterità che vive in me senza che io la riduca a "proprietà".

Vivere in me la trascendenza senza eliminarla mi proietta nella dimensione teleologica della storia personale e comunitaria. La trascendenza in Husserl comprende non solo il telos del mondo ma anche il fondamento di esso, aprendo, così, aduna ipotesi creazionista. Questa dimensione " altra" è ripresa da Husserl negli ultimi manoscritti inediti, dei quali ci fa memoria Angela Ales Bello.

"Man mano che l'analisi fenomenologica procede s'illumina il mio passato originario; più mi muovo verso un telos più capisco e presentifico la mia natura originaria. Sono tra due orizzonti infiniti : il passato e il futuro. 


\section{Aoristo)))))}

International Journal of Phenomenology, Hermeneutics and Metaphysics

Nell'esplicitazione di questi orizzonti mi trovo limitato nella temporalità . L'ego del cogito è nel tempo presente [...] come viva presenza, come lebendige Gegenwart. L'apparire dell'essere è nel tempo. Ma per ogni apparire c'è qualcosa di nascosto, un'infinita potenzialità non attuata" (ALES BELLO, 1985, p.32). Nella vita intenzionale, come presenza, c'è la totalità infinita, implicita e non esplicita. In quanto nascosta la presenza originaria è presenza infinita, originaria che contiene tutte le possibili esplicitazioni che possono uscire da essa. Se la presenza nascosta è infinita, la presenza come evidenza è finita.

La presenza è sempre relazione perché ha in sé il passato come ritenzione e il futuro come protenzione.

La presenza nascosta è anche presenza che si rivela a noi stessi: noi siamo questa rivelazione, siamo la certezza evidente di noi a noi stessi.

Attraverso la riduzione dell' esperienza trascendentale alla sfera di ciò che vivo, come appartenente a me, scopro il "proprio" e "l'alter ego"; scopro di vivere come mia la trascendenza senza eliminare la trascendenza.

Nella indagine di Husserl è implicito un interrogativo: se io parto dalla mia presenza, come posso trovare la presenza altrui? A tal proposito, l'analisi husserliana dei modi di coscienza, risulta illuminante.

Così recita un testo husserliano tradotto da Angela Ales Bello: “Comprendo la coscienza estranea sulla base dell'entropatia. [...] si tratta di un processo analogico, ed esso presuppone come riempimento finale la possibilità di una percezione adeguata. Dunque deve sussistere la possibilità che il mio avere empatia trovi il suo riempimento in una percezione dei vissuti dell'altro. Così come la credenza relativa ad una immagine trova il suo riempimento se io vedo la cosa in se stessa [...]. Ora, in questo caso, la rappresentazione di un'immagine presuppone una cosa concreta, che ha il suo posto nel mondo[...]. Ma che cosa accade a proposito dell'entropatia? [...]Essa non si accorda con una conferma ottenuta percettivamente. Se ascriviamo a Dio (come coscienza che tutto comprende) la capacità di penetrare nella coscienza degli altri, ciò è possibile solo a condizione che l'Essere di Dio comprenda in sé ogni altro essere assoluto. Per Lui non si avrebbe nessuna contraddizione (non avrebbe bisogno di nessun riempimento) poiché Dio non avrebbe bisogno , ad es. ,un campo visivo, ma tanti quanti sono le coscienze assolute [...].Dio vede la cosa da un lato con la mia coscienza- e "contemporaneamente" da un altro lato - con la coscienza di un altro " (ALES BELLO, 1985, p. 68-70).

Nel rapporto tra immanenza e trascendenza, Husserl, utilizzando lo schema monadico di Leibniz, definisce Dio come Monade Somma, ed è proprio l'indagine sulla intersoggettività che, come ricerca costitutiva, ha il compito di descrivere il senso oggettivo che si costituisce come senso originario dell'essere realizzantesi "nel come della realtà soggettiva ".

Nel Ms sulla Monadologia-c ,1930, troviamo la seguente affermazione del Nostro: "La costituzione del mondo è costituzione di sempre più alta umanità, la quale è consapevole del suo essere proprio e vero e assume la forma di un tutto che liberamente costituisce se stesso avendo come scopo la ragione, e la forma della perfezione. 


\section{Aoristo)))))}

International Journal of Phenomenology, Hermeneutics and Metaphysics

Dio non è semplicemente la totalità delle monadi, ma è l'Entelechia che si trova nella totalità come idea del telos dello sviluppo infinito, quello dell'umanità come assoluta ragione, intesa come ciò che regola necessariamente l'essere monadico, e lo regola secondo una propria libera decisione. Questo , in quanto intersoggettivo, è un processo necessariamente allargantesi, senza il quale, nonostante episodi di decadenza, l'essere universale non può neppure esistere “(ALES BELLO, 1985, p.77).

La soggettività trascendentale, intesa nella sua struttura intersoggettiva, consente di individuare nella teleologia, la forma originaria (Ur-form ).

Perché la tensione dell'umanità verso il perfezionamento sia viva, questa forma originaria deve essere costantemente riscoperta.

Seguiamo Husserl che, a tal proposito scrive nel Ms E III 2: "L'universale direzione dell'io è verso il bene [...] la prima e originaria creazione del mio io, nel volere me stesso, è nel rivolgermi universalmente ai beni, all'idea dei beni pratici, e indirizzarmi a ciò che vale, di presentarmi come l'incarnazione (la migliore possibile) del valore. [...] La concreta soggettività primordiale include tutti i modi di coscienza, quindi anche quelli empatici e quelli comprendenti l'esprimersi delle persone nelle cose. Soggettività che racchiude i modi di coscienza nei quali sono posti come validi: la natura, lo spirito (inteso) in ogni senso (umano ed animale), come spirito oggettivo in quanto cultura, come essere spirituale in quanto famiglia, gruppo, stato, popolo, umanità. [...] In tutti i modi di coscienza, e nei suoi modi di considerazione, si trova che essi portano qualcosa a" validità", e ciò che è valido in essi appartiene esso stesso inseparabilmente a tutti questi modi di coscienza, ad un insieme esperito ed esperibile. [...] Ora considero che nella ricerca regressiva (Rückfrage) si dà la struttura originaria nel suo mutamento della hyle originaria con le cinestesi originarie, i sentimenti originari, gli istinti originari [...] (e) si trova nel "fatto "che il materiale originario si fonde in una forma di unità, che è la forma essenziale della mondanità. Perciò, appare preindicata la costituzione di tutto il mondo (.). Per me [...] la teleologia scopribile, trascendentale, risiede nel fatto" (ALES BELLO, 1985, p.100).

Il Nostro nel contesto di questa analisi fa emergere un carattere unitario, che conseguentemente alla "validità" come "forma di unità" (mondana) si presenta come una "disposizione durevole", essa è l'amore.

E se primariamente si presenta come amore personale, realizzante un piacere ed una dedizione legate all'amato, con il progredire dell'analisi, si estende al prossimo (l'amore senza riserve del Cristo) e alla comunità intera: è l'amore etico (ethische Liebe) “[...] che consiste nel prendersi cura amorevolmente dell'altro, del suo essere e divenire etico, è una disposizione verso gli altri, verso i gruppi umani, verso l'intera umanità [...] L'amore cristiano è in primo luogo solo amore. Ma esso è legato al tendere verso la realizzazione della comunità d'amore in una dimensione più ampia possibile [...]. (Così) dalla costituzione di sé della soggettività trascendentale come essere orientato all'infinito verso la perfezione e verso la vera autoconservazione, deriva la necessità della presa di coscienza di sé in gradi sempre più alti. [...] Il processo teleologico dell'essere dell'intersoggettività trascendentale, porta in sé

$$
\begin{array}{r}
\text { Maria Concetta Rípullo } \\
\text { Toledo, v. 4, } n^{\cdot} 2(2021) \text { p. 153 162 }
\end{array}
$$




\section{Aoristo)))))}

International Journal of Phenomenology, Hermeneutics and Metaphysics

un'universale volontà di vita, dapprima oscura nei singoli soggetti. [...] Nel suo sviluppo essa diviene esplicita in alcuni soggetti, così l'uomo si desta nel suo orizzonte trascendentale, si desta in lui l'orizzonte della genuina umanità.

Questa idea si trova in lui come formata preontologicamente. Attraverso la riflessione riceve forma scientifica nella ontologia, e in quanto idea ontologica può diventare trainante per la volontà, che, in tal modo, ha il suo fine esplicito, come meta dello scopo di tutti gli scopi intersoggettivi, ovvero l'idea della perfezione all'infinito.

La volontà assoluta universale che vive in tutte le soggettività trascendentali e che rende possibile l'essere individuale-concreto della soggettività, è la volontà divina, la quale presuppone l'intersoggettività (non nel senso che quest'ultima precede la prima) ma come strati strutturali, senza i quali questa volontà non può essere concreta" (ALES BELLO, 1985, p.98).

Lo sviluppo morale dell'umanità in Husserl è originato da un fondamento:

a) l'amore;

b) dall'evoluzione di esso: l'amore etico;

c) dalla realizzazione di un fine: la realizzazione della genuina umanità.

Queste linee guida dello sviluppo storico-provvidenzialistico si snodano nella indagine rigorosamente fenomenologica, chiaramente significata dalla affermazione del Maestro di seguito riportata: "Se io riconosco ciò in quanto fenomenologo, allora $\mathrm{mi}$ rendo conto di essere nello sviluppo universale e questo è un fatto "(ALES BELLO, 1985, p.96).

\section{REFERENZE}

ALES BELLO, A. Husserl sul problema di Dio. Roma: Studium, 1985.

HUSSERL, E. Idee per una fenomenologia pura e una filosofia fenomenologica. (Ed.) V. Costa. Torino: Einaudi, 2002. . Introduzione all'etica. Lezioni del semestre estivo (1920/1924). (Ed.) F. S. Trincia; Trad. N. Zippel. Bari: Laterza, 2019. . Meditazioni Cartesiane (Ed.) F. Costa. Milano: Bompiani, 1994. . Ricerche logiche. v. II. (Ed.) G. Piana. Milano: il Saggiatore, 1968.

$\overline{\mathrm{PACl}}, \mathrm{E}$. Tempo e Verità nella fenomenologia di Husserl. Bari: Laterza, 1961.

Inserito: 15 giugno 2021

Accettato: 15 luglio 2021 